\title{
Frozen Sections in Pleural Pathology: A Valuable Tool
}

\author{
Georgia Karpathiou $^{a}$ Marios Froudarakis ${ }^{b}$ Fabien Forest $^{a} \quad$ Mousa Mobarki $^{a}$ \\ Arnaud Patoir ${ }^{c}$ Pierre Gay ${ }^{b}$ Jean Michel Vergnon ${ }^{b}$ Olivier Tiffet ${ }^{c}$ \\ Michel Peoc'h ${ }^{a}$ \\ Departments of a Pathology, ${ }^{b}$ Pneumonology and Thoracic Oncology, and ${ }^{\mathrm{C}}$ Thoracic Surgery, North Hospital, \\ University Hospital of Saint-Étienne, Saint-Étienne, France
}

For editorial comment see p. 16

\section{Keywords}

Pleurodesis · Mesothelioma · Pleural metastasis .

Thoracoscopy

\begin{abstract}
Background: Knowledge of pleural malignancy can lead to immediate pleurodesis during thoracoscopy. However, the accuracy of pleural frozen sections is largely unknown. $\mathbf{O b}$ jectives: To investigate the accuracy of frozen sections in pleural tumor pathology. Methods: A total of 156 frozen pleural sections performed with the question of malignancy were retrospectively reviewed. The original frozen sections were compared to the permanent section slides which were considered as the gold standard. The influence of the following parameters on the frozen section response was evaluated: specimen size, clinical information, as well as the processing by a specialized pulmonary pathologist or not. The reasons of discrepancies were categorized as sampling errors or interpretation errors. Results: Frozen sections made up $16.4 \%$ of 951 pleural biopsies performed in the same time period. Accurate diagnosis was feasible in $92.3 \%$ of the cases. There were 7 (4.5\%) deferred (inconclusive) cases and 5 (3.2\%) discrepant cases. Sensitivity of the method was $96.26 \%$, specificity $97.87 \%$, the positive predictive value was
\end{abstract}

\section{KARGER}

(C) 2017 S. Karger AG, Basel

E-Mail karger@karger.com

www.karger.com/res
$99.04 \%$, and the negative predictive value was $92 \%$. There was no association between the responses given during frozen section and specimen size, clinical information, or the evaluation by a specialized pulmonary pathologist. Four of the 12 cases were sampling errors, while 8 cases were interpretation errors mostly made in the absence of fat tissue invasion. Thus, paucicellular lesions without prominent invasion - fat invasion or haphazardly invading cellular proliferation - were those posing most of the difficulties during frozen section. Conclusions: Frozen sections are a highly accurate tool in pleural pathology. Thus, they can be used when an immediate pleurodesis is requested.

(c) 2017 S. Karger AG, Basel

\section{Introduction}

Pleural nodules or thickening are common findings of pleural malignancy either primary or metastatic. When facing with a pleural effusion, preliminary steps to diagnosis classically include clinical history and examination at presentation as well as chest radiography and computed tomography with ultrasound and pleural thoracocentesis [1]. Detecting the cause of a pleural effusion can be difficult, and minimal invasive techniques such as pleural

Georgia Karpathiou, MD, BSc, PhD Department of Pathology University Hospital of Saint-Étienne FR-42055 Cedex2 Saint-Étienne (France) E-Mail gakarpath@yahoo.gr 
fluid thoracocentesis, and blind or imaging-guided biopsy are often insufficient [1]. Many factors exist for negative results in minimal invasive procedures such as the histology of the primary tumor, and the pattern as well as the extent of invasion of the pleural cavity [2]. Therefore, when there is a strong clinical suspicion of malignancy, patients will undergo thoracoscopy, medical or surgical, in order to diagnose the effusion cause and, if malignant, proceed to pleurodesis, the treatment that will prevent fluid recurrence and palliate dyspnea, thus improving patient's quality of life $[1,3]$.

In order to proceed directly to pleurodesis, respiratory physicians or thoracic surgeons performing thoracoscopy need confirmation of malignancy. Immediate response can be achieved by frozen sections, a technique widely used in pathology departments. This could prevent the need of a second thoracoscopy for pleurodesis after the definite pathology report of metastatic tumor or malignant pleural mesothelioma (MPM). Despite pleural frozen section is probably a routine practice in hospitals with an active thoracic team, it has never been studied in terms of accuracy as a technique used in pleural pathology.

Thus, the aim of the study was to determine the accuracy of frozen sections in diagnosing malignancy and the reasons for the discrepancy between frozen section diagnoses and permanent section diagnoses.

\section{Material and Methods}

\section{Study Group}

This is a retrospective study of diagnoses made in all consecutive frozen sections of pleural tissue at the University Hospital of Saint-Étienne, Saint-Étienne, France, during a period of 6 years (2010-2016). The Ethics Committee of the University Hospital of Saint-Étienne approved the study (DC-2015-2489). All frozen sections were originally requested with the question of malignancy. Diagnoses during frozen section were retrieved and compared to the final pathology diagnosis of the same specimen which was considered as the gold standard [4]. Diagnoses during frozen sections were categorized as positive, negative, or inconclusive for the presence of malignancy, in this latter case suggesting to wait until permanent sections are made (deferred cases). We also studied parameters such as the size of the specimen, clinical information given at the time of the frozen section, as well as the processing by a specialized pulmonary pathologist (G.K., F.F.) or not.

\section{Frozen Sections Technique}

When the specimen arrives in its fresh state, macroscopic examination starts with measurement of the biopsy size and describing tissue aspects, which, accompanied by palpation, reveals any nodules. A cytologic sample (touch imprint) is routinely made, followed by a macroscopic section of the most nodular region. If nodules are absent, a section of the most thickened area is made. This section is immediately frozen in the cryostat into a layer of optimal cutting temperature mounting medium. The section cut into the cryostat is rapidly warmed $\left(55-60^{\circ} \mathrm{C}\right)$ and then stained into a toluidine blue bath for few seconds. After coverslip, the section is ready to be examined under the microscope. In all deferred cases, a second section of another region of the specimen has been also examined. In few cases $(n=16)$, a second opinion was asked before submitting the final answer.

\section{Histological Evaluation}

The original frozen sections were analyzed and compared to the conventionally formaldehyde-fixed paraffin-embedded tissue sections (permanent sections). If histological features between the frozen section and all permanent section slides were not the same, then the reason for discrepancy was thought to be a sampling error, meaning that at the time of the frozen section the region sampled and examined was not representative of the lesion, which was only revealed after additional permanent sections were performed. If histological features were the same between frozen section and permanent section slides, then the reason for discrepancy was thought to be an interpretation error [5], meaning that the lesion was actually present at the slide examined during frozen section but it was not correctly interpreted.

The deferred cases were excluded from the analysis of diagnostic accuracy in terms of sensitivity/specificity, positive/negative predictive value and positive/negative likelihood ratio, as in these cases neither a malignant nor a benign diagnosis was made and as such the answer given during frozen section cannot be considered neither as false/true positive nor as false/true negative [4].

As for the definitive section diagnosis, this is based on standard histological criteria [6]. Immunohistochemical study was generally adapted to each case but the antibodies most often used were: CK5/6 (D5/16 B4; Dako, Courtaboeuf, France, 1/600), Calretinin (DC8; Invitrogen, 1/200), D2-40 (Dako), HBME1 (HBME1; Dako, 1/150), WT1 (6F-H2; BD Biosciences), ACE (II-7; Dako, 1/400), CD15 (Carb-3; Dako, 1/200), BerEP4 (Ber-EP4; Dako, 1/50), TTF1 (8G7G3/1; Dako, 1/50), p40 (RP163; Clinisciences, Nanterre, France, 1/75), AE1/AE3 (AE1/AE3; Dako, 1/200), and KL1 (KL1; Beckman-Coulter, Villepinte, France, 1/400). They were used in a panel of at least 2 mesothelioma and 2 carcinoma markers based on the morphology, and continued with subsequent antibodies if the results were not definite. Furthermore, MPM had been systematically reviewed by the French mesothelioma network (MESOPATH) with confirmation by P16 FISH and BAP1 immunohistochemistry in some of these cases.

Cytologic examination prior to thoracoscopy has been performed in our center or from referring centers, but thoracoscopy and frozen sections have been decided in the basis of a strong suspicion of malignancy, even when the answer was negative or inconclusive, or for having tissue for further histologic confirmation and molecular analysis or due to the will to perform pleurodesis, necessitating prior a certain histological diagnosis.

Diagnostic Accuracy

Sensitivity: the probability that a result will be positive when the disease is present $=a /(a+b)$.

Specificity: the probability that a result will be negative when the disease is not present $=d /(c+d)$.
46

Respiration 2017;94:45-51

DOI: $10.1159 / 000474952$
Karpathiou/Froudarakis/Forest/Mobarki/ Patoir/Gay/Vergnon/Tiffet/Peoc'h 
Positive likelihood ratio: the ratio between the probability of a positive test result given the presence of the disease and the probability of a positive test result given the absence of the disease (true positive rate/false positive rate) $=$ Sensitivity/1-Specificity.

Negative likelihood ratio: the ratio between the probability of a negative test result given the presence of the disease and the probability of a negative test result given the absence of the disease (false negative rate/true negative rate) $=1$-Sensitivity/Specificity.

Positive predictive value: probability that the disease is present when the test is positive $=a /(a+c)$.

Negative predictive value: probability that the disease is not present when the test is negative $=d /(b+d)$, where a stands for true positive (cases answered as positive for malignancy during frozen section and proven indeed malignant after permanent sections), b stands for false negative (cases answered as negative for malignancy during frozen section but turn out to be malignant after permanent sections), c stands for false positive (cases answered as positive for malignancy during frozen section but turn out to be nonmalignant in permanent sections), $\mathrm{d}$ stands for true negative (cases answered as negative for malignancy during frozen section and proven indeed nonmalignant in permanent sections).

\section{Statistical Analysis}

Data were analyzed using the StatView software (Abacus Concepts, Berkeley, CA, USA). The relationship between 2 groups was investigated using the $\chi^{2}$ test for categorical data. For all analyses, statistical significance was indicated at a $p$ value of $<0.05$.

\section{Results}

The study group involved 156 frozen sections of the pleura. At the same time period, a total of 951 pleural biopsies have been received; thus, pleural frozen sections were performed in $16.4 \%$ of all pleural biopsies. The characteristics of the study group are presented in Table 1 . Forty-nine specimens were obtained from females and 107 from males, all presenting with recurrent pleural effusions. In 53 cases, a known or highly suspected malignancy of pulmonary $(n=28)$ or other $(n=25)$ origin was mentioned by the thoracoscopist intraoperatively.

During frozen section, a malignancy was confirmed in 97 cases $(62.2 \%)$ and absence of malignancy was found in 52 cases $(33.3 \%)$. Seven cases $(4.5 \%)$ were inconclusive requiring permanent sections for definitive diagnosis (deferred cases). Definitive diagnoses made in conventionally fixed sections included 105 malignancies (67.3\%) and 51 nonmalignant cases $(32.7 \%)$. Of the inconclusive cases, 2 showed no malignancy and in 5 cases malignancy was found in permanent sections. Of the nondeferred cases, 1 case was diagnosed as malignant in frozen section but finally diagnosed as nonspecific pleuritis in permanent sections, and 4 cases were diagnosed as nonmalignant in frozen sections but proved to be malignant in per-

Frozen Sections in Pleural Pathology
Table 1. Study group characteristics $(n=156)$

\begin{tabular}{|c|c|}
\hline Characteristics & \\
\hline \multicolumn{2}{|l|}{ Patient characteristics } \\
\hline Age, years & $70(19-86)$ \\
\hline \multicolumn{2}{|l|}{ Sex } \\
\hline Female & $49(31.4)$ \\
\hline Male & $107(68.6)$ \\
\hline \multicolumn{2}{|c|}{ Clinical information at the time of the frozen section } \\
\hline \multicolumn{2}{|c|}{ Known or highly suspected primary } \\
\hline Lung cancer & $28(18)$ \\
\hline Other & $25(16.1)$ \\
\hline Pleural nodule & $7(4.5)$ \\
\hline Pulmonary nodule & $6(3.8)$ \\
\hline Asbestos exposure & $9(5.8)$ \\
\hline Hemothorax & $1(0.6)$ \\
\hline Tuberculosis & $1(0.6)$ \\
\hline No information & $79(50.6)$ \\
\hline \multicolumn{2}{|l|}{ Tumor characteristics } \\
\hline \multicolumn{2}{|c|}{ Diagnosis in frozen sections } \\
\hline Malignant & $97(62.2)$ \\
\hline Benign & $52(33.3)$ \\
\hline Inconclusive & $7(4.5)$ \\
\hline \multicolumn{2}{|c|}{ Diagnosis in conventionally fixed sections } \\
\hline Malignant & $105(67.3)$ \\
\hline Benign & $51(32.7)$ \\
\hline \multicolumn{2}{|c|}{ Diagnosis in conventionally fixed sections } \\
\hline \multicolumn{2}{|c|}{ Metastasis } \\
\hline Lung cancer & $32(20.5)$ \\
\hline Other primary & $40(25.7)$ \\
\hline \multicolumn{2}{|l|}{ MPM } \\
\hline Epithelioid & $24(15.5)$ \\
\hline Sarcomatoid & $6(3.8)$ \\
\hline Biphasic & $1(0.6)$ \\
\hline Other tumors & $3(2.2)$ \\
\hline Reactive condition & $50(32.1)$ \\
\hline Specimen size, $\mathrm{cm}$ & $0.5-17.4$ \\
\hline
\end{tabular}

Values are given as median (range) or as $n(\%)$.

manent sections (Fig. 1). Thus, an accurate diagnosis was feasible in $92.3 \%$ of the cases. Sensitivity of the method, after excluding deferred cases [4], was $95.6 \%$, specificity was $97.96 \%$, the positive predictive value was $98.96 \%$, and the negative predictive value was $92.31 \%$ (Table 2).

No associations between the responses given during frozen section and the size of the specimen $(p=0.9)$ or the clinical information given at the time of the frozen section ( $p=0.1$ ) were found. A specialized pulmonary pathologist has examined the frozen section in 73 cases and a nonspecialized (4 different pathologists) in 83 cases: no differences were found between the responses given $(p=$ $0.5)$. 
Fig. 1. Cases included in the study.

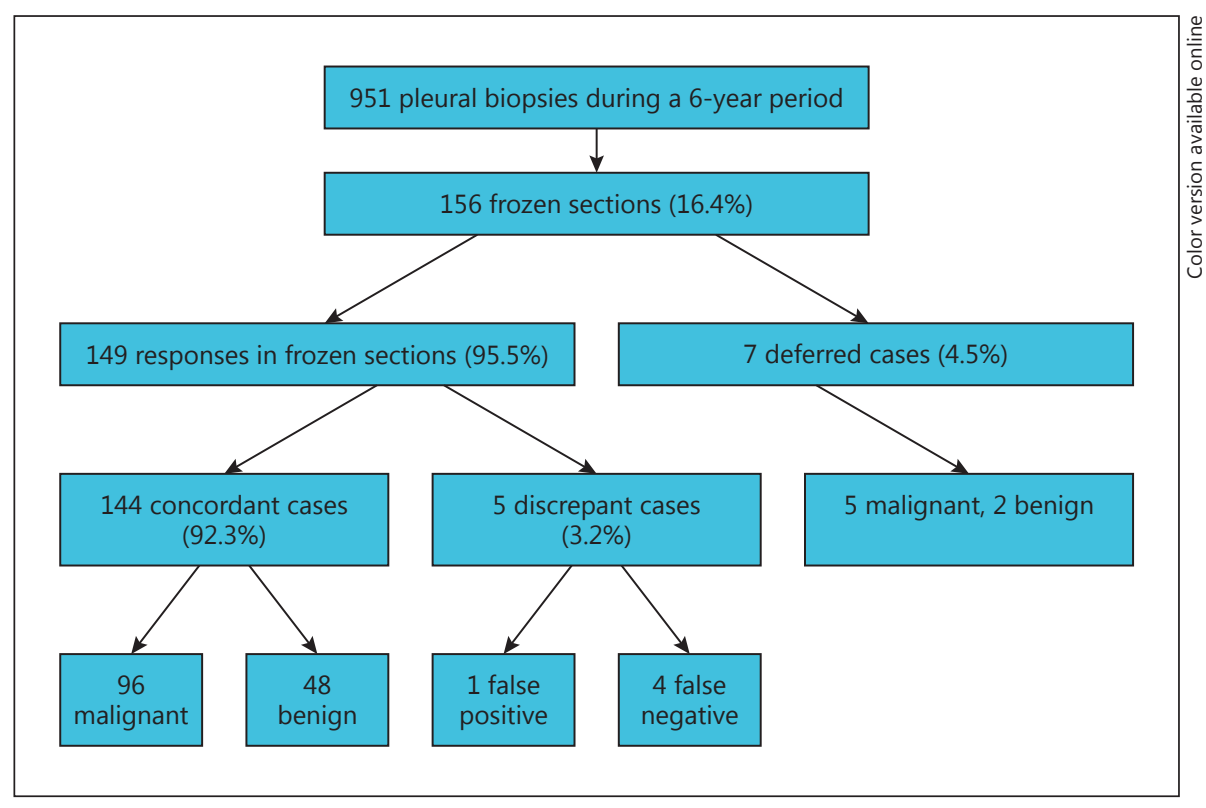

Table 2. Efficacy of frozen section in diagnosing malignancy

\begin{tabular}{lcc}
\hline Accuracy & $92.3 \%$ & \\
Deferred cases & $4.5 \%$ & \\
Discrepant cases & $3.2 \%$ & \\
Statistic & & \\
$\quad$ Sensitivity & $96.26 \%$ & $90.70-98.97$ \\
$\quad$ Specificity & $97.87 \%$ & $88.71-99.95$ \\
$\quad$ Positive likelihood ratio & 45.24 & $6.51-314.64$ \\
Negative likelihood ratio & 0.04 & $0.01-0.10$ \\
Disease prevalence & $69.48 \%$ & $61.56-76.64$ \\
Positive predictive value & $99.04 \%$ & $93.68-99.86$ \\
Negative predictive value & $92.00 \%$ & $81.46-96.79$ \\
\hline
\end{tabular}

Values in the right column are $95 \%$ CI.

The case that was diagnosed as positive during frozen section but was finally negative for malignancy was a 75 -year-old male with a known lung cancer. Frozen pleural section was moderately cellular with lymphocytes, fibroblasts, and vascular proliferation and thus misinterpreted as positive. Of the 4 cases diagnosed during frozen section as negative but proven malignant in permanent sections, 3 were sampling errors, as malignancy was confirmed in other sections; all 3 samples were of paucicellular tumors ( 1 squamous cell carcinoma, 1 adenocarcinoma, and 1 bisphasic MPM) producing an important fibrosing reaction without macroscopically evident nodules, so as to choose the best area for frozen section. The fourth was an interpretation error, as glandular structures were recognized but they were not abundant neither invasive into fat, so as to permit a definitive diagnosis of malignancy (Fig. 2c, d). Of the 7 deferred cases, 4 were mesotheliomas ( 2 epithelioid, 1 sarcomatoid, and 1 desmoplastic), 2 were metastatic carcinomas and 1 was a pleuritis. In the desmoplastic mesothelioma, only collagen without cellular proliferation was recognized; in the other 3 MPM, there was an evident proliferation but no invasion of fat tissue was recognized, withholding the diagnosis at frozen section. Similarly, the 2 metastases contained cellular proliferation without prominent invasion, so the diagnosis was withhold (Fig. 2a, b). Finally, a pleural biopsy rich in inflammatory cells and large endothelial cells was considered suspicious and waiting was recommended until permanent sections which revealed pleuritis (Fig. 2e, f). A typically positive frozen section causing no diagnostic problems is shown in Figure 3.

Thus, 4 of the 12 cases (deferred/discrepant) were sampling errors, while 8 were interpretation errors mostly made in the absence of fat tissue invasion.

\section{Discussion}

Frozen sections were first introduced in 1891 by William Henry Welch of the John Hopkins Hospital and established as a practical technique in 1905 by Louis B. Wilson of the Mayo Clinic [7]. The technique is based on
48

Respiration 2017;94:45-51 DOI: $10.1159 / 000474952$
Karpathiou/Froudarakis/Forest/Mobarki/ Patoir/Gay/Vergnon/Tiffet/Peoc'h 

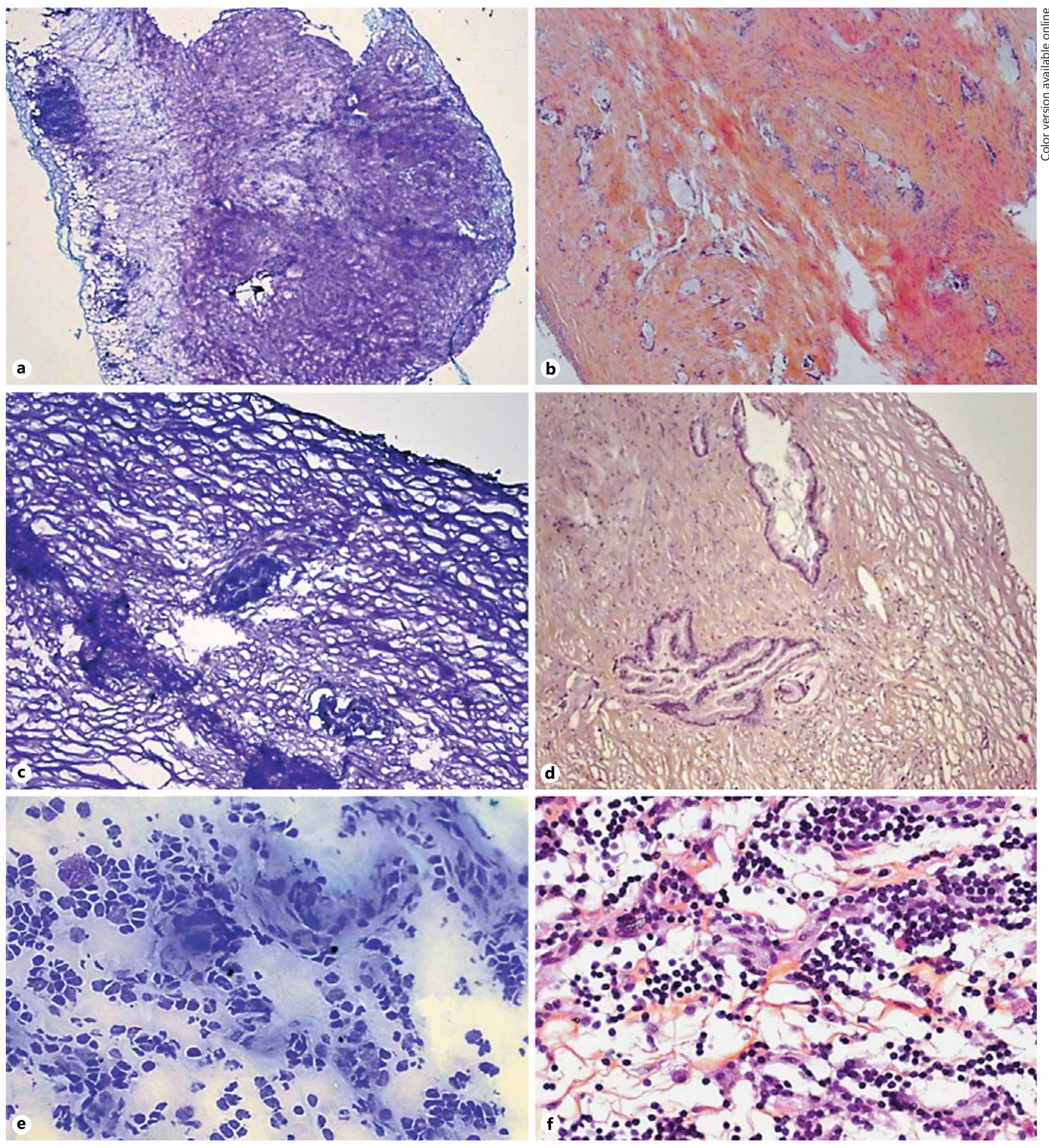

Fig. 2. Representative microphotographs of cases in which the correct diagnosis has not been achieved in frozen section. a Frozen section (toluidin blue $\times 25$ ) of a deferred case in which few atypical glandular structure were found. Neither fat invasion nor important cellularity/nodularity is found to ascertain the diagnosis. b HES section $(\times 50)$ revealing TTF1-positive glandular structures compatible

with lung cancer metastasis. c Frozen section (toluidin blue $\times 100$ ) showing very few glandular structures responding as negative for malignancy. d HES section $(\times 100)$ showing a similarly paucicellular mucus-secreting metastatic tumor. e Frozen section (toluidin blue $\times 400$ ) showing lymphoid cells and some large atypical cells considered suspicious. $\mathbf{f}$ HES section $(\times 400)$ showing pleuritis. 

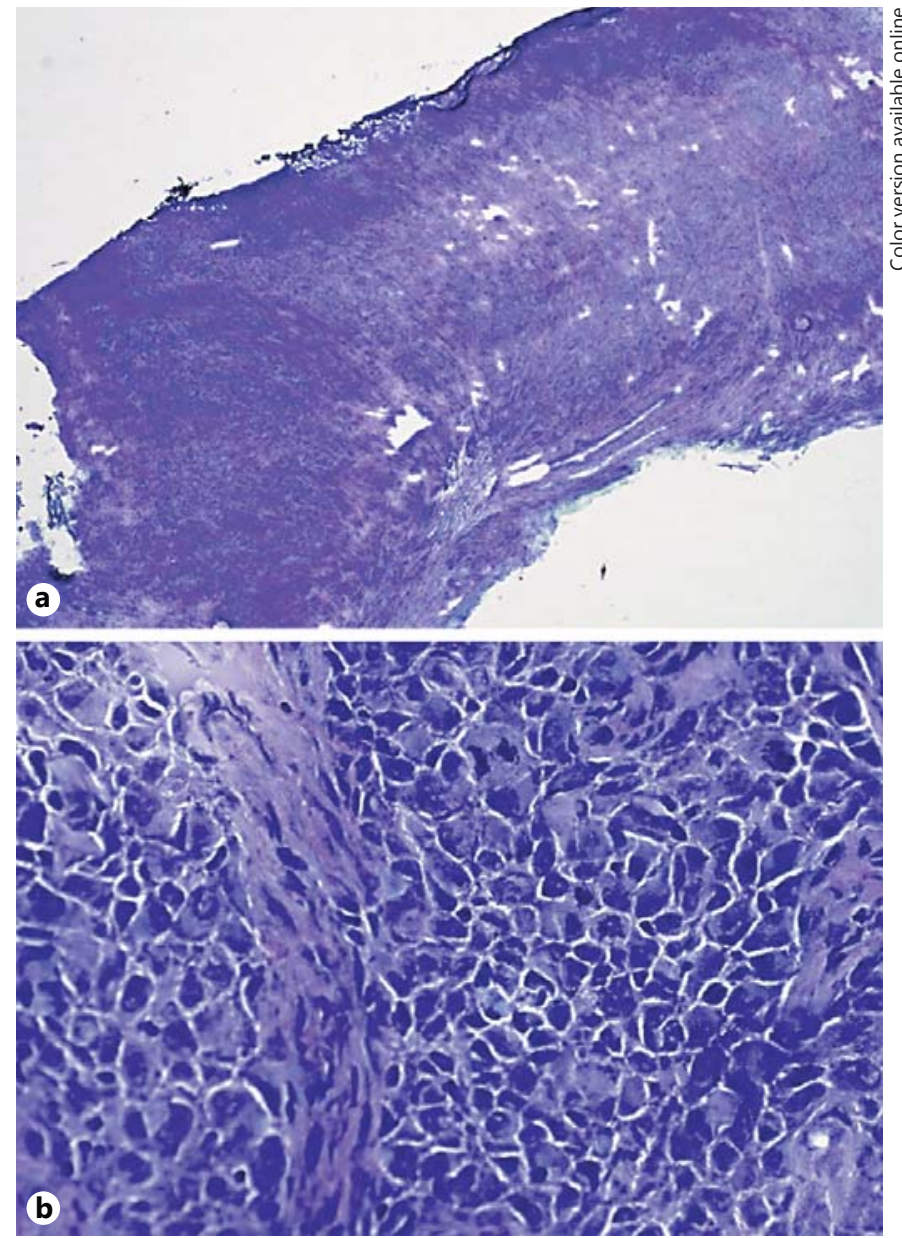

Fig. 3. Representative microphotographs of a malignant tumor easily recognized during frozen section. $\mathbf{a}(\times 25)$ and $\mathbf{b}(\times 400)$ show frozen sections (toluidin blue) of a tumor (finally sarcomatoid MPM) where cellularity, atypia, and mitoses are sufficient to diagnose malignancy even in the absence of fat invasion. HES, hematoxylin, eosin, saffron.

rapidly freezing the tissue in order that it is be able to produce microsections and, after a quick staining method, to perform a microscopic analysis. Generally, it is used when the immediate response of the pathologist is going to change the intraoperative decision or to ascertain material adequacy for further investigation. In thoracic pathology, it is used to confirm pulmonary malignancy to proceed to surgery when a preoperative histological diagnosis was impossible to obtain, to assess bronchial margins, to evaluate lymph nodes for intraoperative staging reasons, to immediately define the nature of a mediastinal mass, and to confirm pleural malignancy to proceed to immediate pleurodesis. Frequently, ade- quacy of the sample for further techniques is the reason of a thoracic frozen section. Knowing immediately the nature of a sample is very important in the molecular pathology era in order to best schedule all necessary examinations, like tissue banking, immunohistochemistry demands, and molecular analysis [8].

Although often used to evaluate pleural disease, the accuracy of pleural frozen sections has been never studied, with the exception of 1 report examining 10 malignant and 10 benign cases by frozen section of pleural tissue obtained by medical thoracoscopy [9]. Three of the cases were deferred and 2 showed discrepancy in diagnosis ( 2 cases assessed as benign were finally malignant); thus, the accuracy was $75 \%$. In this study, we showed that frozen sections are used in $16.4 \%$ of pleural biopsies made, an important number for the routine practice of a pathology department regarding educative, cost, and time consequences but also thoracic disease departments for initiation of immediate pleurodesis. Most important, it is a highly accurate technique with a sensitivity and a specificity for detecting malignancy of 96.26 and $97.87 \%$, respectively, highlighting the usefulness of the technique in the clinical decision-making of pleurodesis. When a frozen section is positive for malignancy, there is a probability of $99 \%$ for a malignancy to be present in the definitive diagnosis.

Pleurodesis aims to develop symphysis between parietal and visceral pleura, which will obliterate the pleural space and finally restrain fluid from accumulating [10, 11]. Talc is currently the best sclerosing agent shown to be effective, safe, and cheap and providing long-lasting results [3]. Pleurodesis is achieved mostly by talc insufflation into the pleural cavity performed under thoracoscopy [3]. Thoracoscopic pleurodesis is reserved for patients with a good health status and a relatively long life expectancy, in whom a histological diagnosis and anatomic and molecular staging of the disease, lead to changes in the treatment strategy [12]. In order to perform this technique, a prior diagnosis should have been made as after pleurodesis almost no tissue can be obtained. Frozen section diagnosis is important as it gives the possibility to perform thoracoscopic pleurodesis at the same settings, and therefore, spares the patient from a second unnecessary procedure $[12,13]$.

As for the main difficulties rendering an immediate diagnosis by frozen section impossible, in one quarter of the case, there were sampling errors originating mostly from paucicellular tumors not generating evident nodules. In these cases, pathologists did not find any tumoral nodules during macroscopic examination of the speci-
50

Respiration 2017;94:45-51

DOI: $10.1159 / 000474952$
Karpathiou/Froudarakis/Forest/Mobarki/ Patoir/Gay/Vergnon/Tiffet/Peoc'h 
men to choose the correct area for the frozen section. Similarly, during thoracoscopy, sampling errors do occur. These are mostly due to either adhesions or a fibrinous layer on the pleura hampering extensive investigation of the pleural cavity [14]. In the rest of our cases, the difficulty was mostly found in the interpretation of biopsies harboring a cellular proliferation, either fusiform or epithelioid, that was indeed evident but did not invade the underlying fat to secure the diagnosis. The same cases posed difficulties even during the definite diagnosis. It is true that regarding the differential diagnosis of MPM from a reactive condition, either mesothelial hyperplasia or fibrosing pleuritis, fat invasion is the most important criterion, along with nodular architecture and prominent cellularity invading vertically or without organization the whole thickness of the fibrosing pleura [6]. Recently, p16 FISH and BAP1 immunohistochemistry have been proven useful for this differentiation [6]. Thus, during frozen section, moderately cellular lesions without prominent invasion - defined as fat invasion or haphazardly invading cellular proliferation -, are those to pose most of the difficulties.

Our study has certain limitations. First, it is a retrospective study and some clinical information, such as side effects and complications of the technique are missing, although this was not the goal of the current study. In addition, factors for the final diagnosis of both frozen or definite histological diagnosis, such as consensus after discussion, might be missing. However, we were able to report second opinions when necessary, as it is included in our pathology report. Another limitation is the lack of patient follow-up, especially those with a histological diagnosis of benign pleural effusion. However, we believe that the conditions described in the current study largely reflect the reality in most departments dealing with pleural pathology.

In conclusion, pleural frozen sections are a valuable and accurate tool in immediately diagnosing malignancy, permitting further techniques like pleurodesis in the same settings and allowing for the correct sample preparation. During intraoperative consultation, pleurodesis should be withhold when there is no definitive answer from the pathologist or when no malignancy is found in frozen section, but it can be directly performed when malignancy is found in frozen section.

\section{Acknowledgment}

The authors would like to thank Mr. Philippe Cosmo from the Tumorothèque/Centre de Ressources Biologiques de CHU SaintEtienne (BRIF no. BB-0033-00041) for his assistance during the preparation of the manuscript.

\section{Disclosure Statement}

The authors have no conflicts of interest to disclose.

\section{References}

1 Froudarakis ME: Diagnostic work-up of pleural effusions. Respiration 2008;75:4-13.

2 Anevlavis S, Kouliatsis G, Sotiriou I, et al: Prognostic factors in patients presenting with pleural effusion revealing malignancy. Respiration 2014;87:311-316.

3 Froudarakis M: Should we really compare indwelling pleural catheters to any pleurodesis? Respiration 2014;88:18-21.

4 Goyal R, Zhu B, Parimi V, Lin X, Rohan SM: Diagnostic accuracy of frozen-section analysis of cancer-containing bladder transurethral resection specimens for the presence of muscularis propria invasion. J Clin Pathol 2014; 67:562-565.
5 Yeh Y-C, Nitadori J, Kadota K, et al: Using frozen section to identify histological patterns in stage I lung adenocarcinoma of $\leq 3 \mathrm{~cm}$ : accuracy and interobserver agreement. Histopathology 2015;66:922-938.

6 Karpathiou G, Stefanou D, Froudarakis ME: Pleural neoplastic pathology. Respir Med 2015;109:931-943.

7 Gal AA: The centennial anniversary of the frozen section technique at the Mayo Clinic. Arch Pathol Lab Med 2005;129:1532-1535.

8 Karpathiou G, Batistatou A, Forest F, Clemenson A, Peoc'h M: Basic molecular pathology and cytogenetics for practicing pathologists: correlation with morphology and with a focus on aspects of diagnostic or therapeutic utility. Adv Anat Pathol 2016;23:368-380.

9 Fielding D, Hopkins P, Serisier D: Frozen section of pleural biopsies at medical thoracoscopy assists in correctly identifying benign disease. Respirology 2005;10:636-642.
10 Rodriguez-Panadero F, Montes-Worboys A: Mechanisms of pleurodesis. Respiration 2012;83:91-98.

11 Froudarakis ME, Klimathianaki M, Pougounias M: Systemic inflammatory reaction after thoracoscopic talc poudrage. Chest 2006;129: 356-361.

12 Froudarakis M: Pleural diseases in the molecular era - time for more answers: introduction. Respiration 2012;83:2-4.

13 Rodriguez-Panadero F: Medical thoracoscopy. Respiration 2008;76:363-372.

14 Janssen J, Ramlal S, Mravunac M: The longterm follow-up of exudative pleural effusion after nondiagnostic thoracoscopy. J Bronchol 2004;11:169-174. 